\title{
THE FORCE OF A TINY SYNTHETIC MACHINE
}

¿ Tiziana Svaldo-Lanero and Anne-Sophie Duwez - DOI: 10.1051/epn/2013303

— University of Liège, Department of Chemistry, Sart - Tilman B6a, 4000 Liège, Belgium - asduwez@ulg.ac.be

In order to function, living organisms use an amazing number of molecular machines. These machines are essential in controlling and performing numerous biological functions, like muscle contraction or intracellular transport. They are able to rectify random thermal motion to generate force and carry out macroscopic tasks. This ability has inspired attempts to create synthetic machines exhibiting control over motion to perform work.

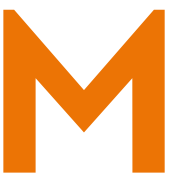

olecular biological machines have inspired chemists who, for some years now, have been synthesising systems capable of mimicking the natural world [1-3] by producing mechanical work that can manifest itself at the microscopic or macroscopic scale. Examples include the transport of a droplet up an inclined surface against gravity [4], the rotation of submillimetre objects on a substrate [5], or the deflection of a microcantilever [6]. A molecular machine is a molecular complex composed of two parts which can move with respect to one another in response to a stimulus (light, electrons, a concentration gradient,...), the net result being mechanical work. This intramolecular motion is capable of generating a considerable directional force. Despite remarkable advances in recent years, there is still a lack of a deep understanding of how to harness sub-molecular motion to generate a useful response to perform physical tasks.

Rotaxanes are prototypical synthetic molecular machine systems. They are made of a molecular ring threaded onto a molecular axle [3]. (Fig. 1). The thread bears one or more recognition sites called stations, onto which the ring can bind through intra-molecular bonds according to its affinities. The chemical environment also forces the ring to preferentially remain on one of the stations (where it can make the strongest bonds, this site being thermodynamically favoured). Rotaxanes can behave as molecular shuttles: the ring can be translocated from one binding site on the thread to the other one through biased Brownian motion in response to an external trigger [3]. The collective dynamics of such systems have been studied with increasing structural sensitivity and temporal precision [7]. In contrast, much of the exquisite and detailed

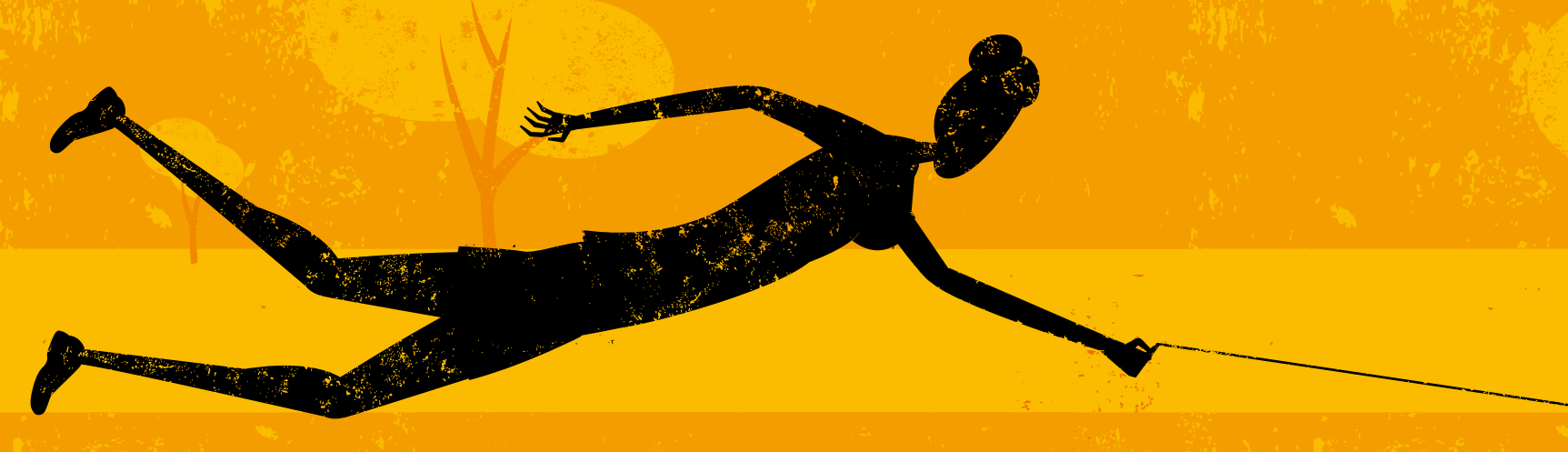


information about how biomolecular machines operate has been gleaned from direct measurements made on single molecules with manipulation techniques like optical tweezers or force-clamp atomic force microscopy (AFM). Such measurements have shown that single biological machines are able to generate forces against loads of 5-60 $\mathrm{pN}$, and have highlighted many details about their mechanical properties [8].AFM-based single molecule force spectroscopy is a tool able to monitor mechanical forces with sub-nanometer resolution and has been widely used to investigate molecular-level processes $[9,10]$.

Although single macromolecules, such as proteins and synthetic polymers, have been widely manipulated and studied by single-molecule force spectroscopy, implementing this technique on smaller molecules remains a major challenge. We have designed a hydrogen-bonded rotaxane-based molecular shuttle with a tether attached to the ring to track its motion by an AFM cantilever. The [2] rotaxane consists of a ring mechanically locked onto a thread by two bulky groups situated at either end of the axle (Fig. 2 ). The thread bears two sites, each of which can bind to the ring through up to four hydrogen bonds [11]. The ring predominantly resides over one of the two sites, the occupancy ratio being higher than 95:5. A polymeric chain, suitable for binding to an AFM tip, was attached to the ring. Specific groups were introduced to enable the grafting of the rotaxane onto a gold surface. The rotaxane-tether molecules were grafted onto substrates in a dilute distribution (isolated molecules).

We used the cantilever of an AFM microscope to catch the tether, then apply a mechanical load to the ring of the rotaxane and follow its motion [12]. The caught molecules were stretched in a controlled manner by moving the tip away from the substrate at a fixed pulling rate, and the force-extension profiles were measured (Fig. 3). This is very random, just like fishing: often we don't catch anything

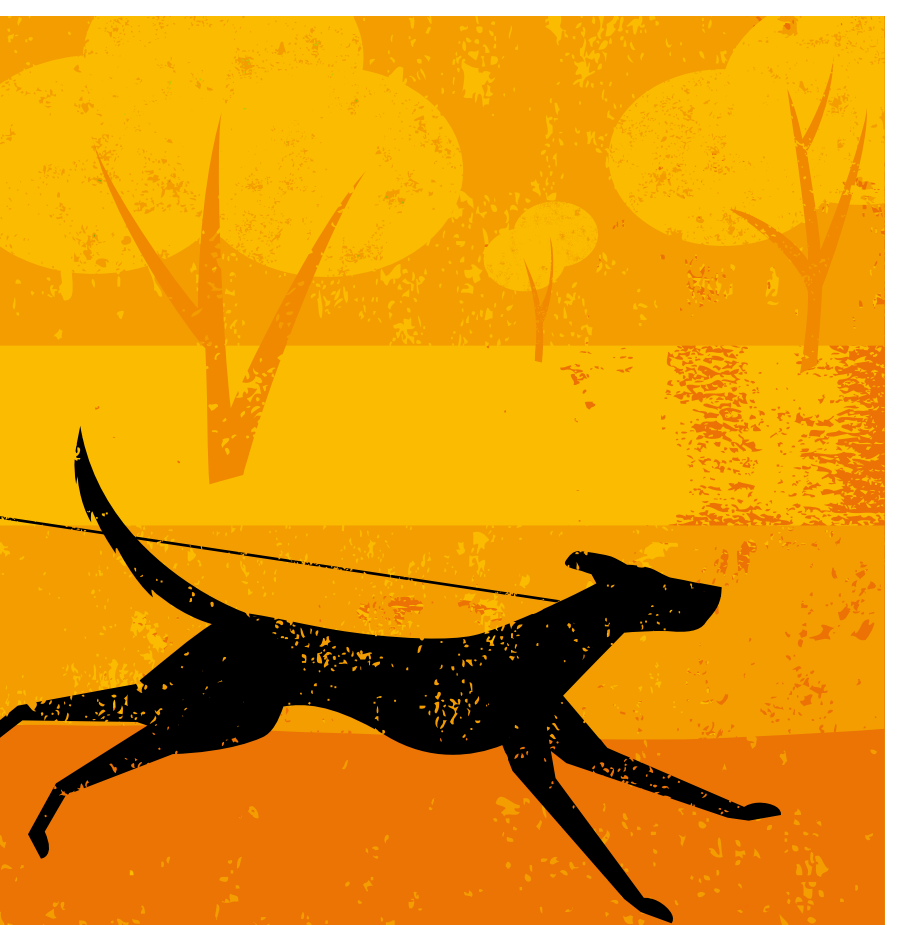

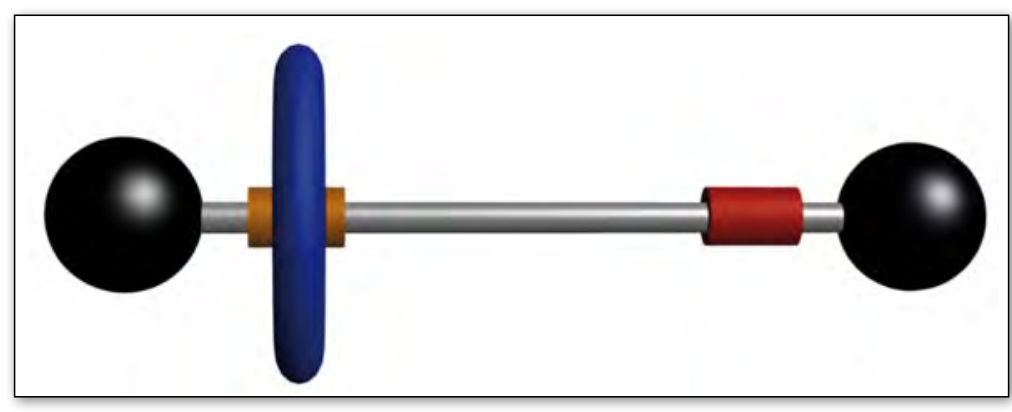

but sometimes we catch the tether of a rotaxane, and pull it up. If the force exerted on the tether is stronger than the hydrogen bonds that bind the ring to its preferred site, the ring detaches from its station. It is then pulled along the axle, away from its most stable binding site. We noticed that the ring of the rotaxane was capable of travelling back to this site, in the direction opposite to the force exerted on it by the cantilever, against a mechanical load of $30 \mathrm{pN}$, thus delivering mechanical work. If we trap the ring and then slightly decrease the force on the tether while maintaining the pulling, the ring is capable of pulling more strongly to recover its preferred position [12].

This exploit is the result of eight long years of research and collaboration with Professor D.A. Leigh's team (University of Edinburgh, currently at the University of Manchester) who has synthesised the rotaxane, and Professor C.-A. Fustin's team (Université catholique de Louvain) who connected the polymeric tether.

For the first time, a quantitative measurement of the work done by a single synthetic molecule less than $5 \mathrm{~nm}$ long has been achieved. It shows that the biased Brownian motions caused by thermal energy can be harnessed within a single synthetic small molecule, to generate significant directional forces similar in magnitude to those generated by natural molecular machines, which are a lot bigger. The results also show that in certain conditions (loading rate, solvent, etc.), this synthetic nanomachine is capable of using and converting $100 \%$ of the energy available in the molecule in the form of binding energy to perform mechanical work. The study of systems at the single molecule level has considerable importance on a fundamental level. Indeed, the laws of physics and chemistry which govern the macroscopic world are no longer necessarily valid at the molecular level. The principles of thermodynamics describe processes of energy exchange (work and heat) of macroscopic systems with their environment. In a general way, in macroscopic systems, the average behaviour is reproducible and the fluctuations are of little importance. When the dimensions of the system become nanoscopic, the fluctuations can give rise to significant deviations with regard to the average behaviour. An individual molecule can, for example, extract energy (which comes from thermal energy) from its environment to perform a mechanical work higher than the energy available in the molecule. This is inconceivable $\triangle \mathrm{FIG}$. 1: Illustration of a [2]rotaxane with one thread (grey) and two stations (orange and red), one ring (blue) and two bulky end-stoppers at either end to prevent the ring from dethreading. $\varangle$ A simplistic representation of the molecular machine experiment. $A$ woman holds a dog on a leash and exerts a pulling force on the leash to hold the dog back, but the dog decides to run, carrying the woman along, even if the latter continues to dog therefore exerts the mechanical load that the woman represents and which continues to pull in the opposite direction. Cistockphoto pull on the leash. The a higher force against 


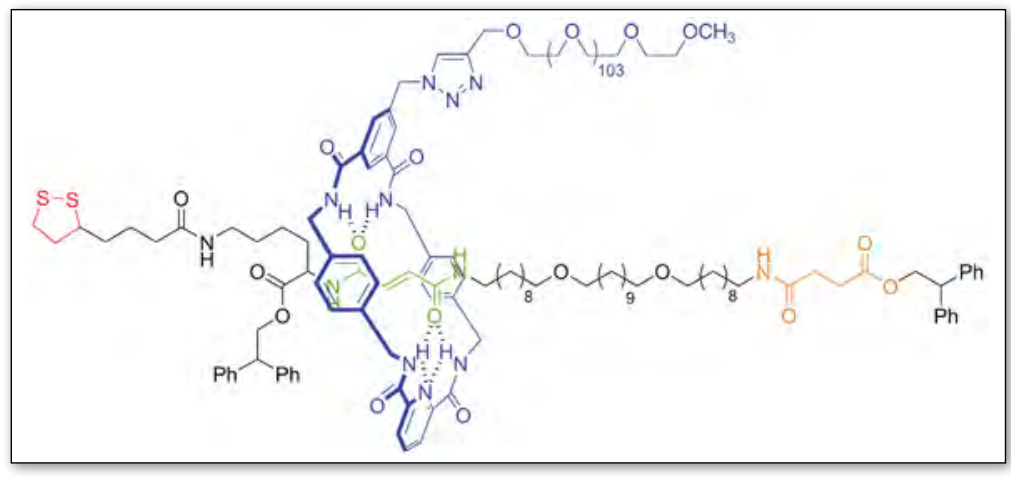

for a set of billions of molecules because they all have to do it at the same time, which is statistically impossible. Thus, well-known concepts of physics and chemistry must be redefined or improved in order to reconcile the nanoscopic and macroscopic worlds. It is from this perspective that for several years, theoretical physicists have established fluctuation theorems. These theorems reconcile what we observe on a large scale and for a large number of molecules in equilibrium with the data obtained for a single molecule (or a small number of molecules) in non-equilibrium conditions. For the first time, we have shown that it is possible to apply modern theories of non-equilibrium statistical mechanics to AFM force measurements.

Studying the movements in a single molecule of less than 5 nanometres long and measuring the work it can perform is a real breakthrough. If manipulations at the molecular level have been known for fifteen years, so far it was a matter of measuring the movements within biological molecules which are a thousand times bigger than a rotaxane molecule.

\section{About the Authors}

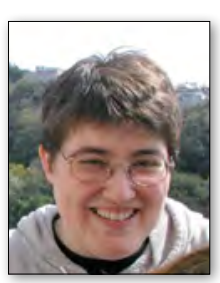

Tiziana Svaldo-Lanero studied Physics at the University of Genoa, Italy, where she also obtained her $\mathrm{PhD}$ in Biotechnology in 2008. In 2008-2009, she was Post-doc at Duke University, Durham, USA. Since 2009 she is Post-doc in the group of Professor A.-S. Duwez at the University of Liège, Belgium.

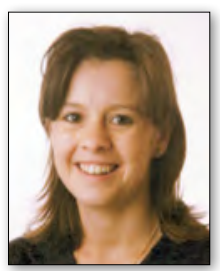

Anne-Sophie Duwez received her $\mathrm{PhD}$ in Chemistry from the University of $\mathrm{Na}$ mur,Belgium. After a post-doc at the MaxPlanck Institute in Mainz, Germany and a senior scientist position at the Université catholique de Louvain, she took up the Chair of Chemistry at Surfaces at the University of Liège in 2006 and set up a new lab. She is currently professor of surface chemistry and nanotechnology. Her research interests focus on the development of AFM-based techniques to manipulate single molecules. They include the investigation of mechanochemical processes in bio- and synthetic systems and the design of single molecule devices.
4 FIG. 2. Chemical structure of the rotaxane molecule, synthesised by the group of D.A. Leigh, UK. The rotaxane consists of a benzylic amide molecular ring (in blue) mechanically locked onto an axle by bulky diphenylethyl ester groups situated at either end. The axle bears a fumaramide group (in green) and a succinic amide-ester group (in orange), either of which can act as a binding site for the ring through up to four intercomponent hydrogen bonds. The affinity of the ring for the fumaramide site is much higher than for the succinic amide-ester site, so that the fumaramide:succinic amide-ester occupancy ratio is higher than 95:5. Next to the fumaramide binding site, a disulfide group (in red) was introduced to enable the grafting of the molecule onto gold substrates. A $4600 M_{n}$ PE0 tether (in blue) is attached to the ring in order to link the molecule to the AFM probe and track the motion of the ring along the axle.

\section{References}

[1] M. Schliwa (ed.), Molecular Motors (Wiley-VCH, Weinheim, 2003).

[2] K. Kinbara \& T. Aida, Chem. Rev. 105, 1377 (2005).

[3] E.R. Kay, D. A. Leigh \& F. Zerbetto, Angew. Chem. Int. Ed. 46, 72 (2007).

[4] J. Berná, D.A. Leigh, M. Lubomska, S.M. Mendoza, E.M. Perez, P. Rudolf, G. Teobaldi, \& F. Zerbetto, Nature Mater. 4, 704 (2005).

[5] R. Eelkema, M.M. Pollard, J. Vicario, N. Katsonis, B.S. Ramon, C.W.M. Bastiaansen, D.J. Broer \& B.L. Feringa, Nature 440, 163 (2006).

[6] Y. Liu, A.H. Flood, P.A. Bonvallet, S.A. Vignon, B.H. Northrop, H.R. Tseng, J.O. Jeppesen, T.J. Huang, B. Brough, M. Baller, S. Magonov, S.D. Solares, W.A. Goddard, C.-M. Ho \& J.F. Stoddart, J. Am. Chem. Soc. 127, 9745 (2005).

[7] M.R. Panman, P. Bodis, D.J. Shaw, B.H. Bakker, A.C. Newton, E.R. Kay, A.M. Brouwer, W.J. Buma, D.A. Leigh \& S. Woutersen, Science 328, 1255 (2010).

[8] Single-molecule theme, Special issue, Annu. Rev. Biochem. 77, 45-228 (2008).

[9] E. Evans, Annu. Rev. Biophys. Biomol. Struct. 30, 105 (2001).

[10] J. Liang \& J.M. Fernández, ACS Nano 3, 1628 (2009).

[11] A. Altieri, G. Bottari, F. Dehez, D.A. Leigh, J.K.Y. Wong \& F. Zerbetto, Angew. Chem. Int. Ed. 42, 2296 (2003).

[12] P. Lussis, T. Svaldo-Lanero, A. Bertocco, C.-A. Fustin, D.A. Leigh \& A.-S. Duwez, Nature Nanotech. 6, 553 (2011).

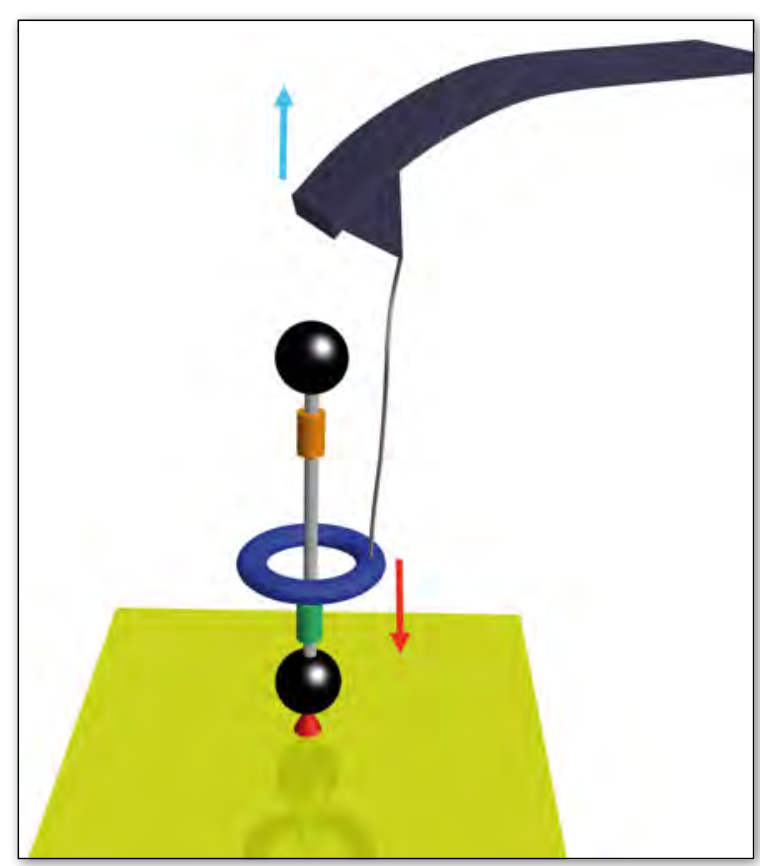

